\title{
Ultrasonic velocity and attenuation anisotropy of shales, Whitby, United Kingdom
}

\author{
Alimzhan Zhubayev ${ }^{1}$, Maartje E. Houben², David M. J. Smeulders ${ }^{3}$, and Auke Barnhoorn ${ }^{4}$
}

\begin{abstract}
We have conducted ultrasonic experiments, between 0.3 and $1 \mathrm{MHz}$, to measure velocity and attenuation $\left(Q^{-1}\right)$ anisotropy of $\mathrm{P}$ - and S-waves in dry Whitby Mudstone samples as a function of stress. We found the degree of anisotropy to be as large as $70 \%$ for velocity and attenuation. The sensitivity of P-wave anisotropy change with applied stress is more conspicuous than for S-waves. The closure of large aspect-ratio pores (and/or micro cracks) seems to be a dominant mechanism controlling the change of anisotropy. Generally, the highest attenuation is perceived for samples that have their bed layering perpendicular $\left(90^{\circ}\right)$ to the wave path. The observed attenuation in the samples is partly due to the scattering on the different layers, and it is partly due to the intrinsic attenuation. Changes in attenuation due to crack closure during the loading stage of the experiment are an indication of the intrinsic attenuation. The remaining attenuation can then be attributed to the layer scattering. Finally, the changes in attenuation anisotropy with applied stress are more dynamic with respect to changes in velocity anisotropy, supporting the validity of a higher sensitivity of attenuation to rock property changes.
\end{abstract}

\section{INTRODUCTION}

Shales are often highly anisotropic. This intrinsic anisotropy is the result of many factors such as partial alignment of platy clay particles, layering, microcracks, kerogen inclusions, and low-aspect-ratio pores (Sayers, 1994, 2013). Shale anisotropy significantly affects seismic wave propagation through shale layers in active seismic surveys, or it influences fracture propagation directions during stimulation of unconventional shale gas reservoirs. As such, shale anisotropy is of great interest to hydrocarbon exploration and production.

Previous attempts to measure seismic anisotropy in shales have been very limited and were primarily devoted to shale velocities and shale velocity anisotropies measured at laboratory scale (Jones and Wang, 1981; Yin, 1992; Vernik and Lin, 1997; Hornby, 1998; Wang, 2002). However, measurements of attenuation anisotropy, despite being very important for the interpretation of rock physical properties of shales, have been very limited (Yin, 1992; Best et al., 2007; Piane et al., 2014).

Attenuation of seismic waves is very sensitive to pore fluid and rock properties, such as permeability, porosity, and the presence of fractures (Pyrak-Nolte et al., 1990; Rubino et al., 2014). Seismic attenuation analyses can be a key seismic attribute in quantitative rock characterization (Mavko et al., 2009). Due to anisotropy, seismic attenuation in shales often depends on the propagation direction. Knowledge of the attenuation anisotropy can potentially be linked to the anisotropic permeability of shales (Rasolofosaon and Zinszner, 2002), its fluid/gas saturation, and fracture orientations (Chichinina et al., 2006; Carcione et al., 2012). This information is of great importance for successful downhole, crosswell, and surface seismic data interpretation. However, the very fragile and water-phobic nature of shales makes sample preparation more complicated, and thus measuring the wave attenuation in shales is also complex. In this paper, we report new anisotropy data (velocity and attenuation) obtained from reasonably dry Whitby Mudstone samples by conducting ultrasonic measurements during stress-controlled experiments. The measured anisotropy data may potentially be used for understanding and/or validating seismic attenuation and can be of much use in new theoretical rock model developments.

We first discuss the Whitby Mudstone Formation sample collection and the chosen strategy for specimen preparation. The experimental setup for recordings at ultrasonic frequencies is briefly

Manuscript received by the Editor 4 April 2015; revised manuscript received 10 July 2015; published online 8 December 2015.

${ }^{1}$ Formerly Delft University of Technology, Department of Geoscience and Engineering, Delft, The Netherlands; presently NAM-Shell Projects and Technology, Assen, The Netherlands. E-mail: alimzhan.zhubayev@ shell.com.

${ }^{2}$ Utrecht University, Faculty of Geosciences, Utrecht, The Netherlands. E-mail: m.e.houben@uu.nl.

${ }^{3}$ Eindhoven University of Technology, Department of Mechanical Engineering, Eindhoven, The Netherlands. E-mail: d.m.j.smeulders@tue.nl.

${ }^{4}$ Delft University of Technology, Department of Geoscience and Engineering, Delft, The Netherlands. E-mail: auke.barnhoorn@tudelft.nl.

(C) 2015 Society of Exploration Geophysicists. All rights reserved. 
explained in the same section. Then, the results of measured velocity and attenuation at ultrasonic frequencies $(0.3-1 \mathrm{MHz})$ in mudstone samples as a function of axial stress and with respect to the bedding plane are presented. Following Thomsen (1986) and Zhu and Tsvankin (2006), the definition of velocity and attenuation anisotropy parameters are given and then used to quantify the anisotropy in our shales.

\section{SAMPLES AND EXPERIMENTAL SETUP}

The Whitby Mudstone Formation (McArthur et al., 2008; Imber et al., 2014) is one possible resource shale for unconventional gas in Northern Europe and currently is of great interest. Shale blocks used in this study were collected during field work at outcrops along the cliff coast north of Whitby, UK, and they are time and depositionally equivalent to the Lower Jurassic Northern European subsurface resource shales (Ghadeer and Macquaker, 2012; Houben et al., 2014).

Collected blocks were prevented from drying by packing them in cling film. Standard laboratory coring techniques were unsuccessful at extracting cylindrical samples out of the mudstone blocks. Highspeed coring resulted quickly into disintegration of the samples. Instead, we first carefully cut rectangular-shaped subsamples out of the larger blocks using a sawing machine without water cooling and subsequently polished the subsamples manually into cylindrical specimens. This more gentle approach ensured that the samples remained intact during the preparation procedure. Porosity and grain density were measured on the cored samples using a helium pycnometer. The porosity was generally less than $5 \%$, whereas solid grain density varied between 2450 and $2650 \mathrm{~kg} / \mathrm{m}^{3}$. The micro$\mathrm{CT}$ analyses and acoustic measurements on shales suggest the validity of (vertical) transverse isotropic (VTI assuming horizontal layering) consideration of our samples. a)

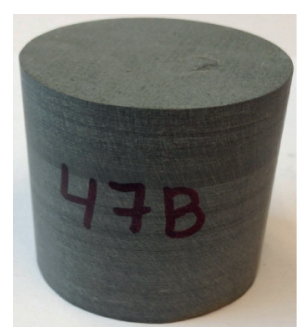

b)

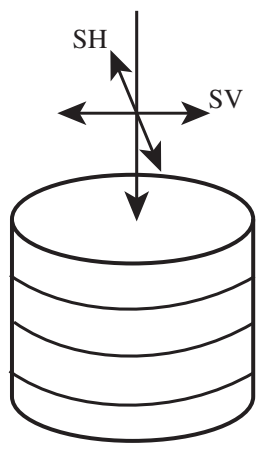

Horizontal sample $\left(90^{\circ}\right)$
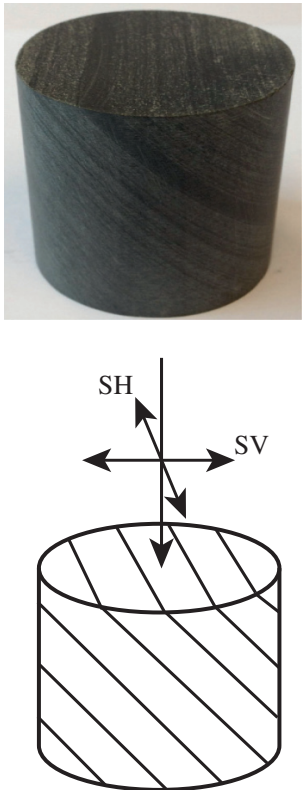

$45^{\circ}$
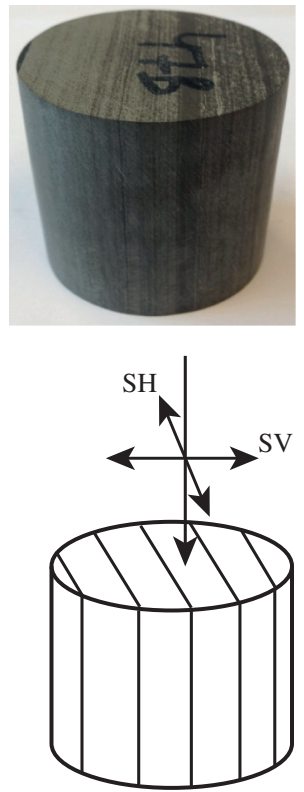

Vertical sample $\left(0^{\circ}\right)$
Figure 1. (a) Photo (WUK47B) and (b) schematic diagram of the three samples. The thin line indicates the bedding plane or lamination. Arrows show the directions of wave propagation and polarization.
To study the ultrasonic anisotropy of shales, we produced three cylindrical samples, $4 \mathrm{~cm}$ in diameter and $4 \mathrm{~cm}$ in length from one shale block. The samples were cut at different angles with respect to the bedding plane. From each shale block, we produced a core perpendicular $\left(90^{\circ}\right)$, parallel $\left(0^{\circ}\right)$, and at a $45^{\circ}$ angle with respect to the bedding plane (see Figure 1). We used three shale blocks (WUK2, WUK47B, and WUK70) in our experiments to study ultrasonic anisotropy, and therefore, nine cores in total were prepared. The three samples originate approximately $1 \mathrm{~m}$ (WUK2), $4 \mathrm{~m}$ (WUK70), and $7 \mathrm{~m}$ (WUK47B) from the base of the Jet Dogger section of the Whitby Mudstone Formation (Houben et al., 2014). All cores were then used to study velocity and attenuation anisotropy at different stress levels by calculating, respectively, $\epsilon, \gamma$, $\delta$ (Thomsen, 1986) and $\epsilon_{Q}, \gamma_{Q}, \delta_{Q}$ (Zhu and Tsvankin, 2006) from ultrasonic measurements (see Appendix A).

Figure 1a and $1 \mathrm{~b}$ shows, respectively, a photo and a schematic diagram of the three samples prepared from block WUK47B. Assuming the validity of (V)TI representation for our shales, five independent velocity-attenuation pairs for complete anisotropy characterization were needed (Thomsen, 1986; Zhu and Tsvankin, 2006).

The shale sample, used for the ultrasonic experiment under axial stress, was placed between two aluminum buffers. Ultrasonic P- or $\mathrm{S}$-wave transducers (one transmitter and one receiver) were coupled on each buffer with a viscous gel and held in contact by springs. The diameter of the transducer is $1 \mathrm{~cm}$, and its central frequency is $1 \mathrm{MHz}$. The acoustic assembly was placed inside an uniaxial loading apparatus. Uniaxial stress experiments were performed at roomtemperature conditions (approximately $20^{\circ} \mathrm{C}$ ) and in the absence of any radial confining pressure. An uniaxial differential stress of up to $30 \mathrm{MPa}$ was applied to the aluminum buffers, and in this way, the Pand S-wave velocity and attenuation of shales were studied. All samples were jacketed with heat-shrink tubing prior to the ultrasonic experiment. A schematic illustration of the experimental setup is shown in Figure 2.

A pulse transmission method (Toksöz et al., 1979; Lucet and Zinszner, 1992) was used to measure the decay of amplitude of the propagating wave in the sample. This technique measures attenuation relative to a reference sample, which has very low attenuation. We have used an aluminum sample as the reference material. Only the response of transmitted $\mathrm{P}$ - and S-wave effects is taken into account. Following Toksöz et al. (1979), the spectral ratio equation can be written as follows:

$$
\ln \left(\frac{A_{1}}{A_{2}}\right)=\left(\beta_{2}-\beta_{1}\right) x f+\ln \left(\frac{G_{1}}{G_{2}}\right),
$$

where $A_{i}$ is the Fourier amplitude; $f$ is the frequency; $x$ is the propagating distance; and $G_{i}$ is the frequency-independent scaling factor due to, e.g., spherical divergence, reflection. Subscripts $i=1$ and $i=2$ correspond to the reference and the mudstone sample, respectively. Finally, $\beta$ encompasses the slope in the spectral ratio method, and it is related to $\mathrm{P}$ - or S-wave attenuation $(1 / Q)$ by 


$$
Q=\frac{\pi}{\beta V},
$$

where $V$ is the ultrasonic $\mathrm{P}$ - or $\mathrm{S}$-wave velocity. This general equation can also be used for directional attenuation calculations using the corresponding estimated spectral amplitude ratio and velocity in the same direction. Velocity and attenuation of the shale are thus measured parallel, perpendicular, and at $45^{\circ}$ to the bedding plane. Source and receiver functions are considered to be the same. An aluminum sample was used as the reference with exactly the same shape and size as the shale samples to be studied. Due to very low attenuation of $Q \approx 150,000$ in aluminum (Zemanek and Rudnick, 1961), $\beta_{1}$ in equation 1 can be approximated to be equal to zero. With $\beta_{1}$ equal to zero, $\beta_{2}$ and thus the attenuation in shale can be calculated using equations 1 and 2 .

Figure 3a shows an example of the recorded signals for aluminum and shale samples using $\mathrm{P}$ source-receiver configuration. By applying a time window around the peak amplitude of the first arrival, an amplitude spectrum was calculated (Figure 3b). The amplitude ratio of the aluminum and shale signals is shown in Figure 3c. Finally, calculating the slope of the best-fit line (the bold line in Figure $3 \mathrm{c})$, one can get the attenuation $\left(Q_{\mathrm{P}}^{-1}\right)$ value of the shale sample according to equations 1 and 2 . The typical frequency range for spectral-ratio fitting was between 0.3 and $1 \mathrm{MHz}$.

Following equation 2, the absolute error of $Q^{-1}$ was calculated using the following formula:

$$
\begin{aligned}
\pm d\left(Q^{-1}\right) & = \pm \frac{1}{\pi} \frac{\partial\left(Q^{-1}\right)}{\partial \beta} d \beta \pm \frac{1}{\pi} \frac{\partial\left(Q^{-1}\right)}{\partial V} d V \\
& = \pm \frac{V}{\pi} d \beta \pm \frac{\beta}{\pi} d V
\end{aligned}
$$

where $d \beta$ and $d V$ are the absolute errors of the slope estimate of the spectral ratio-frequency plot (see Figure $3 \mathrm{c}$ ) and the absolute error of the measured $\mathrm{P}$ - or S-wave velocity, respectively.

The ultrasonic velocity was measured from the first $\mathrm{P}$ - or S-wave arrival time and the height of the sample. The sample shortening and the two aluminum buffers' shortening due to the applied differential stress were taken into account in seismic velocity calculation. The methods to measure seismic velocity and attenuation provide relatively simple yet stable and accurate measurements, and it is a valid approach for comparative purposes.

\section{VELOCITY AND ATTENUATION MEASUREMENTS AS A FUNCTION OF AXIAL STRESS}

To check the repeatability (or stability) of our experiment, the results of the loading experiment were compared with those from the unloading experiment. They were found to be in good agreement with each other, and the results for the horizontal sample $\left(90^{\circ}\right)$ prepared from block WUK47B are shown in Figure 4. Note that this sample was prepared separately to demonstrate the consistency between the loading and unloading experiment only and was excluded from the anisotropy investigation.

Absolute errors in the velocity and attenuation estimates were calculated and were found to be no larger than $3 \%$ and $12 \%$, respectively. An indication of the data quality and the uncertainty can also be easily appreciated by comparison of the scatterplots in Figure 4 (compare the black and white circles at different axial stresses). The estimation of velocity (and thereby attenuation) from first-wave arrivals was corrected for the shortening of the aluminum and shale samples due to increased applied stresses. The maximum aluminum shortening was $0.1 \mathrm{~mm}$ at $40 \mathrm{MPa}$ axial stress.

Figure 5 shows the $\mathrm{P}$ - and $\mathrm{S}$-wave velocity and attenuation measurements as a function of axial stress for three different orientations of cylindrical samples with respect to the bedding and for three dif-

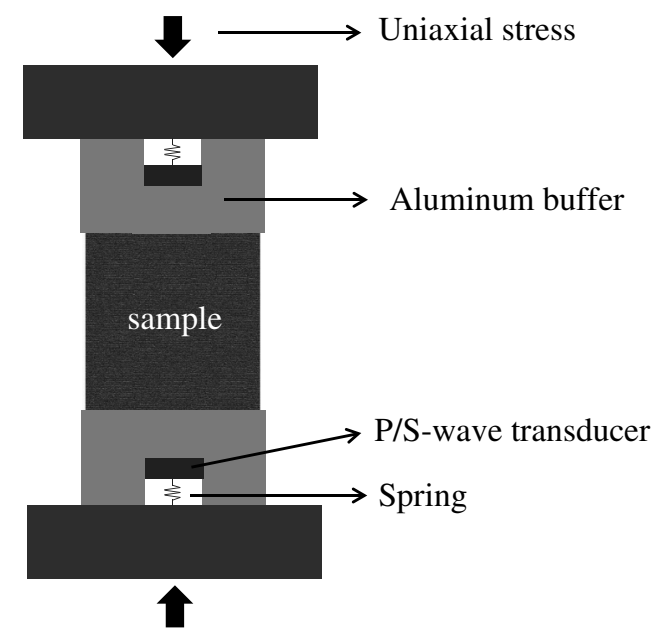

Figure 2. Experimental setup used to measure P- and S-wave velocity and attenuation at different stress conditions.
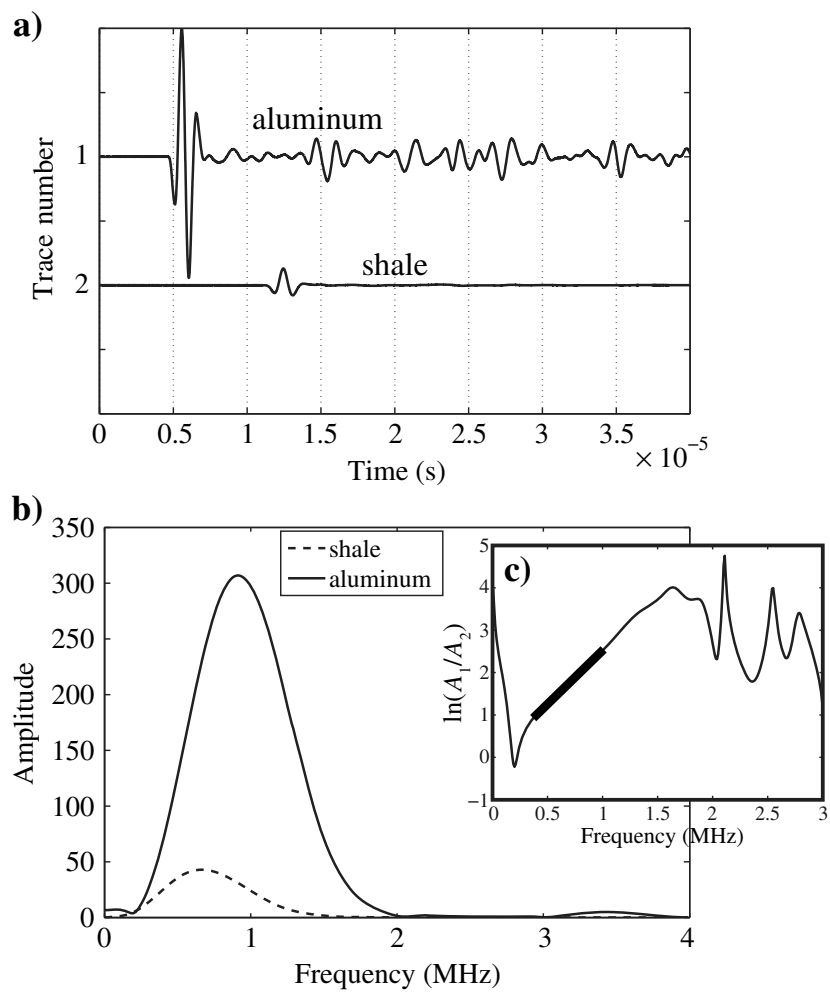

Figure 3. Recorded signals for (a) reference (aluminum) and shale samples and (b) their Fourier spectra. (c) The natural logarithm of the spectral ratio as a function of frequency. 
Figure 4. P- and S-wave velocity and attenuation measurements as a function of axial stress. Stress is perpendicular to the layering.
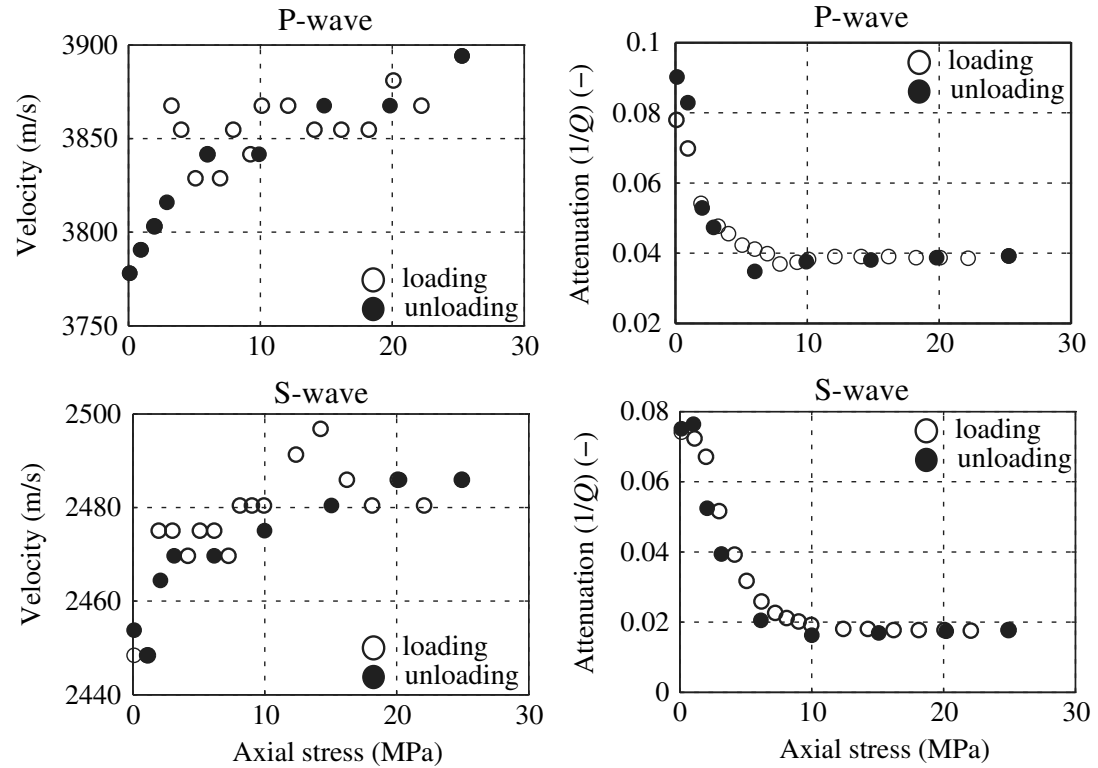

a)
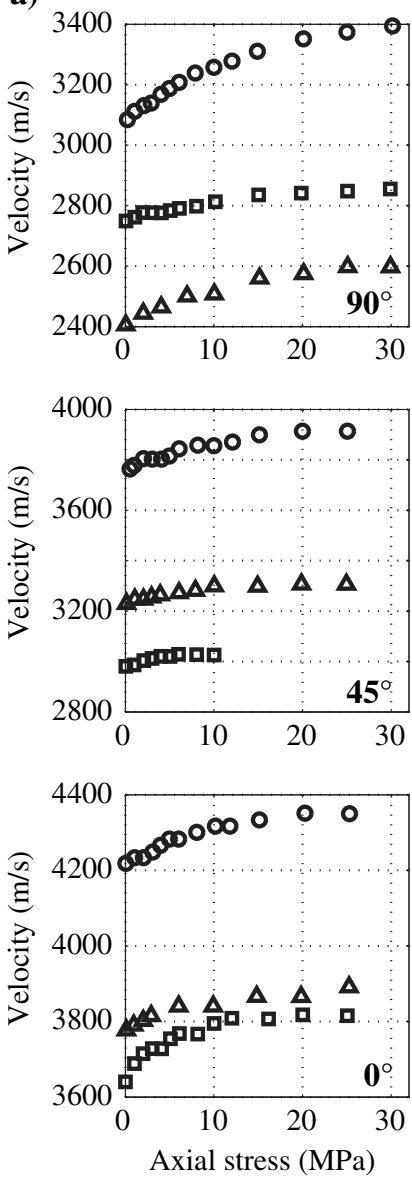
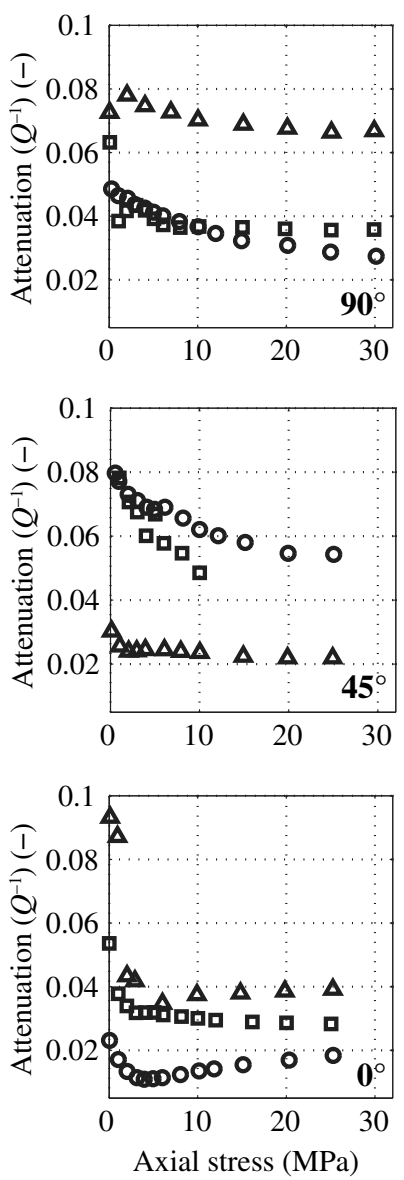

b)
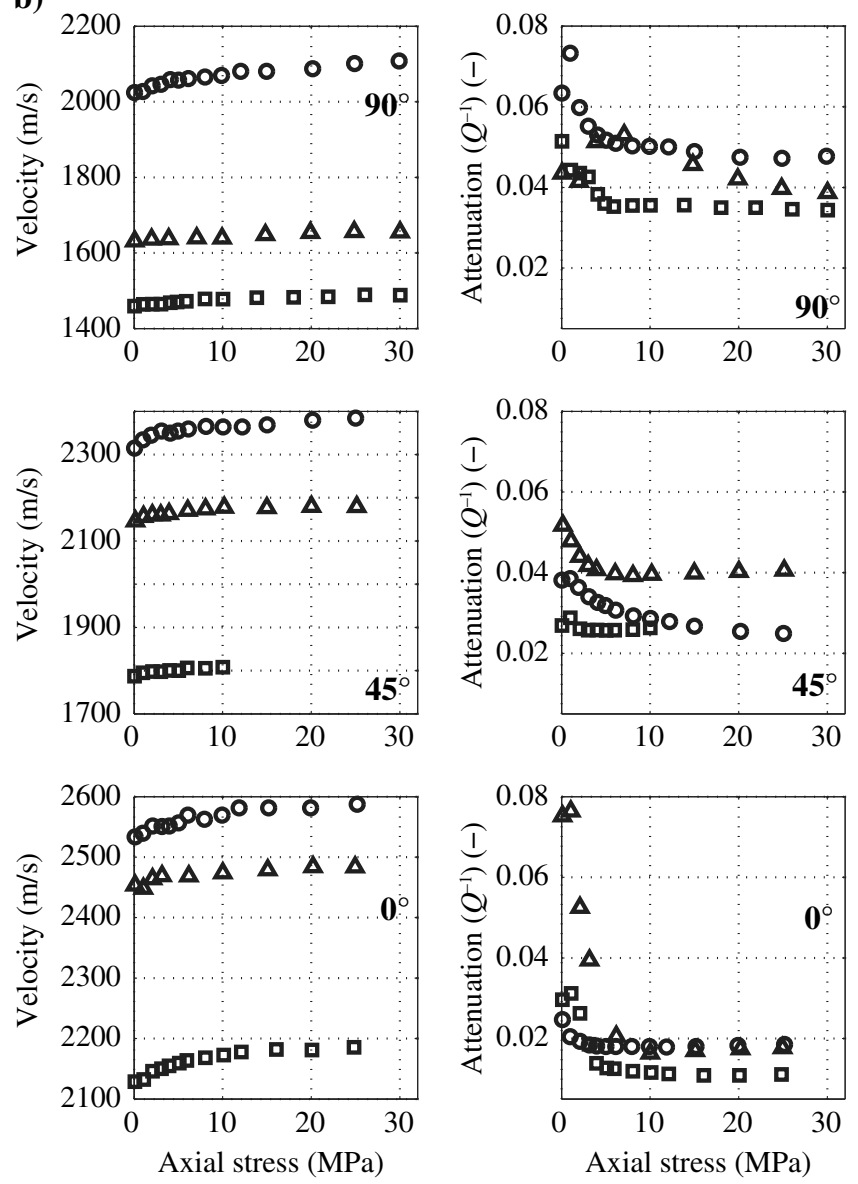

Figure 5. (a) P- and (b) S-wave velocity and attenuation measurements as a function of axial stress in blocks WUK2 (triangles), WUK47B (circles), and WUK70 (squares). The upper, mid, and the bottom rows correspond to the cylindrical samples perpendicular $\left(90^{\circ}\right), 45^{\circ}$, and parallel $\left(0^{\circ}\right)$ to the bedding, respectively. The WUK70 sample deformed with the applied stress at $45^{\circ}$ to the bedding only shows measurements up to $10 \mathrm{MPa}$. The $45^{\circ}$ samples are particularly well oriented for failure along the bedding plane. Because we noticed at approximately $10 \mathrm{MPa}$ that failure could occur soon, we refrained from increasing the applied stresses above that level. 
ferent sets of shale blocks. The measured values and corresponding errors are shown in Tables 1-3. From these results, we can see that $\mathrm{P}$ - and $\mathrm{SH}$-wave velocities increase with the applied differential pressure for all our samples. One can also observe that the attenuation decreases with stress, indicating the closure of compliant cracks and pores under pressure. Note that the effect of compliant crack and/or pore closure can also be seen from velocity measurements at different applied stress. This can be readily appreciated by plotting normalized velocities as a function of stress. In Figure 6, we observe that velocities increase as stress increases, but the velocity gradient in the stress domain $(\partial V / \partial \sigma)$ is different for the different orientations at a given stress. Further examinations reveal that the velocity rate change $\left(\partial V_{\mathrm{P}}\left(90^{\circ}\right) / \partial \sigma\right)$ is highest for the $\mathrm{P}$-wave propagating orthogonal to the bedding, whereas $\partial V_{\mathrm{P}}\left(45^{\circ}\right) / \partial \sigma$ and $\partial V_{\mathrm{P}}\left(0^{\circ}\right) / \partial \sigma$ are more or less the same. Clearly, WUK70 has a lesser amount of open microcracks or pores in the original sample. Hence, the velocity increase due to closure is not that profound as in the other two samples. These results are indicative of preferential align- ments of microcracks and shale minerals. Note also that P-wave velocities are more sensitive to pore and microcrack closure than are the $\mathrm{S}$-wave velocities.

\section{ANISOTROPY}

Ultrasonic velocities for P- and S-waves propagating parallel to the bedding are higher than those propagating orthogonal to the bedding plane (see Figure 5). The following inequality is generally observed for velocity in our shale samples:

$$
V_{\mathrm{P} / \mathrm{S}}\left(90^{\circ}\right)<V_{\mathrm{P} / \mathrm{S}}\left(45^{\circ}\right)<V_{\mathrm{P} / \mathrm{S}}\left(0^{\circ}\right) .
$$

Dynamic Young's moduli and Poisson's ratios of our mudstones under different stress conditions were calculated from the measured velocity (see Appendix A). The results shown in Figure 7 reveal that $E_{1}$ is always larger than $E_{3}\left(E_{1}>E_{3}\right)$ and they increase with ap-

Table 1. WUK2: measured $V_{\mathrm{P}}, V_{\mathrm{S}}, Q_{\mathrm{P}}^{-1}$, and $Q_{\mathrm{S}}^{-1}$ as a function of axial stress.

\begin{tabular}{|c|c|c|c|c|c|}
\hline Sample & Axial stress (MPa) & $V_{\mathrm{P}}$ & $V_{\mathrm{S}}$ & $Q_{\mathrm{P}}^{-1}$ & $Q_{\mathrm{S}}^{-1}$ \\
\hline \multirow[t]{9}{*}{ WUK2 $\left(0^{\circ}\right)$} & 0.01 & $3777 \pm 83$ & $2454 \pm 54$ & $0.093 \pm 0.002$ & $0.075 \pm 0.002$ \\
\hline & 1 & $3790 \pm 83$ & $2448 \pm 54$ & $0.087 \pm 0.002$ & $0.076 \pm 0.002$ \\
\hline & 2 & $3803 \pm 84$ & $2464 \pm 54$ & $0.043 \pm 0.001$ & $0.052 \pm 0.002$ \\
\hline & 3 & $3815 \pm 85$ & $2469 \pm 54$ & $0.042 \pm 0.001$ & $0.039 \pm 0.002$ \\
\hline & 6 & $3841 \pm 55$ & $2469 \pm 54$ & $0.035 \pm 0.001$ & $0.021 \pm 0.002$ \\
\hline & 10 & $3841 \pm 85$ & $2474 \pm 54$ & $0.037 \pm 0.001$ & $0.016 \pm 0.002$ \\
\hline & 15 & $3866 \pm 86$ & $2479 \pm 54$ & $0.038 \pm 0.001$ & $0.017 \pm 0.002$ \\
\hline & 20 & $3866 \pm 86$ & $2484 \pm 54$ & $0.038 \pm 0.001$ & $0.017 \pm 0.002$ \\
\hline & 25 & $3891 \pm 86$ & $2484 \pm 54$ & $0.039 \pm 0.001$ & $0.018 \pm 0.002$ \\
\hline \multirow[t]{11}{*}{ WUK2 $\left(45^{\circ}\right)$} & 0.15 & $3230 \pm 66$ & $2146 \pm 34$ & $0.030 \pm 0.001$ & $0.052 \pm 0.003$ \\
\hline & 1 & $3246 \pm 67$ & $2157 \pm 34$ & $0.026 \pm 0.001$ & $0.048 \pm 0.003$ \\
\hline & 2 & $3247 \pm 67$ & $2160 \pm 34$ & $0.024 \pm 0.001$ & $0.044 \pm 0.003$ \\
\hline & 3 & $3255 \pm 67$ & $2160 \pm 34$ & $0.024 \pm 0.001$ & $0.042 \pm 0.003$ \\
\hline & 4 & $3264 \pm 68$ & $2163 \pm 34$ & $0.025 \pm 0.001$ & $0.041 \pm 0.003$ \\
\hline & 6 & $3272 \pm 68$ & $2170 \pm 34$ & $0.024 \pm 0.001$ & $0.040 \pm 0.003$ \\
\hline & 8 & $3281 \pm 68$ & $2174 \pm 34$ & $0.024 \pm 0.001$ & $0.039 \pm 0.003$ \\
\hline & 10 & $3299 \pm 69$ & $2177 \pm 34$ & $0.024 \pm 0.001$ & $0.039 \pm 0.003$ \\
\hline & 15 & $3298 \pm 69$ & $2177 \pm 34$ & $0.022 \pm 0.001$ & $0.040 \pm 0.003$ \\
\hline & 20 & $3306 \pm 69$ & $2180 \pm 34$ & $0.022 \pm 0.001$ & $0.040 \pm 0.003$ \\
\hline & 25 & $3306 \pm 69$ & $2180 \pm 34$ & $0.021 \pm 0.001$ & $0.041 \pm 0.003$ \\
\hline \multirow[t]{9}{*}{ WUK2 $\left(90^{\circ}\right)$} & 0.05 & $2405 \pm 52$ & $1631 \pm 25$ & $0.072 \pm 0.001$ & $0.043 \pm 0.002$ \\
\hline & 2 & $2443 \pm 53$ & $1636 \pm 25$ & $0.078 \pm 0.001$ & $0.041 \pm 0.002$ \\
\hline & 4 & $2464 \pm 54$ & $1637 \pm 25$ & $0.075 \pm 0.001$ & $0.051 \pm 0.002$ \\
\hline & 7 & $2500 \pm 55$ & $1640 \pm 25$ & $0.073 \pm 0.001$ & $0.052 \pm 0.002$ \\
\hline & 10 & $2507 \pm 55$ & $1640 \pm 25$ & $0.070 \pm 0.001$ & $0.051 \pm 0.002$ \\
\hline & 15 & $2560 \pm 56$ & $1648 \pm 25$ & $0.069 \pm 0.001$ & $0.046 \pm 0.002$ \\
\hline & 20 & $2575 \pm 57$ & $1653 \pm 25$ & $0.068 \pm 0.001$ & $0.042 \pm 0.002$ \\
\hline & 25 & $2597 \pm 57$ & $1656 \pm 25$ & $0.066 \pm 0.001$ & $0.040 \pm 0.002$ \\
\hline & 30 & $2597 \pm 57$ & $1655 \pm 25$ & $0.066 \pm 0.001$ & $0.039 \pm 0.002$ \\
\hline
\end{tabular}


plied pressure. An increase of Poisson's ratios with stress was also found, especially for $\nu_{31}$ of blocks WUK2 and WUK47B.

Figure 8 shows the results of $\mathrm{P}$ - and $\mathrm{S}$-wave attenuation measurements as a function of axial stress as were also shown in Figure 5, but in this case, each panel compares attenuation of $\mathrm{P}$ - (or $\mathrm{S}$ )-wave obtained from three cylindrical samples differently orientated with respect to the bedding from a single shale block. Such a representation is more convenient and gives us a better understanding of the degree of attenuation anisotropy. For P- and S-waves, the highest attenuation is perceived for the samples that have the bed layering

Table 2. WUK47B: measured $V_{\mathrm{P}}, V_{\mathrm{S}}, Q_{\mathrm{P}}^{-1}$, and $Q_{\mathrm{S}}^{-1}$ as a function of axial stress.

\begin{tabular}{|c|c|c|c|c|c|}
\hline Sample & Axial stress (MPa) & $V_{\mathrm{P}}$ & $V_{\mathrm{S}}$ & $Q_{\mathrm{P}}^{-1}$ & $Q_{\mathrm{S}}^{-1}$ \\
\hline \multirow[t]{7}{*}{ WUK47B $\left(0^{\circ}\right)$} & 0.06 & $4218 \pm 86$ & $2533 \pm 46$ & $0.023 \pm 0.002$ & $0.025 \pm 0.001$ \\
\hline & 2 & $4233 \pm 87$ & $2551 \pm 46$ & $0.013 \pm 0.002$ & $0.019 \pm 0.001$ \\
\hline & 3 & $4249 \pm 88$ & $2551 \pm 46$ & $0.011 \pm 0.002$ & $0.018 \pm 0.001$ \\
\hline & 6 & $4283 \pm 89$ & $2569 \pm 47$ & $0.011 \pm 0.002$ & $0.018 \pm 0.001$ \\
\hline & 8 & $4299 \pm 90$ & $2563 \pm 47$ & $0.012 \pm 0.002$ & $0.018 \pm 0.001$ \\
\hline & 10 & $4316 \pm 90$ & $2569 \pm 47$ & $0.013 \pm 0.002$ & $0.018 \pm 0.001$ \\
\hline & 12 & $4316 \pm 90$ & $2582 \pm 47$ & $0.014 \pm 0.002$ & $0.018 \pm 0.001$ \\
\hline \multirow[t]{10}{*}{ WUK47B $\left(45^{\circ}\right)$} & 0.52 & $3763 \pm 83$ & $2315 \pm 39$ & $0.079 \pm 0.004$ & $0.038 \pm 0.002$ \\
\hline & 1 & $3777 \pm 83$ & $2334 \pm 40$ & $0.077 \pm 0.004$ & $0.039 \pm 0.002$ \\
\hline & 2 & $3804 \pm 84$ & $2344 \pm 40$ & $0.073 \pm 0.004$ & $0.036 \pm 0.002$ \\
\hline & 3 & $3803 \pm 84$ & $2354 \pm 40$ & $0.071 \pm 0.004$ & $0.034 \pm 0.002$ \\
\hline & 4 & $3802 \pm 84$ & $2349 \pm 40$ & $0.069 \pm 0.004$ & $0.033 \pm 0.002$ \\
\hline & 5 & $3816 \pm 84$ & $2354 \pm 40$ & $0.068 \pm 0.004$ & $0.032 \pm 0.002$ \\
\hline & 6 & $3844 \pm 85$ & $2360 \pm 40$ & $0.069 \pm 0.004$ & $0.031 \pm 0.002$ \\
\hline & 8 & $3858 \pm 85$ & $2364 \pm 41$ & $0.066 \pm 0.004$ & $0.029 \pm 0.002$ \\
\hline & 10 & $3857 \pm 85$ & $2365 \pm 41$ & $0.062 \pm 0.004$ & $0.029 \pm 0.002$ \\
\hline & 12 & $3871 \pm 86$ & $2364 \pm 41$ & $0.060 \pm 0.004$ & $0.028 \pm 0.002$ \\
\hline \multirow{11}{*}{ WUK47B $\left(90^{\circ}\right)$} & 3 & $3140 \pm 64$ & $2046 \pm 41$ & $0.044 \pm 0.001$ & $0.055 \pm 0.001$ \\
\hline & 4 & $3169 \pm 65$ & $2058 \pm 41$ & $0.042 \pm 0.001$ & $0.053 \pm 0.001$ \\
\hline & 5 & $3188 \pm 66$ & $2058 \pm 42$ & $0.041 \pm 0.001$ & $0.052 \pm 0.001$ \\
\hline & 6 & $3208 \pm 66$ & $2062 \pm 42$ & $0.040 \pm 0.001$ & $0.051 \pm 0.001$ \\
\hline & 8 & $3238 \pm 67$ & $2065 \pm 42$ & $0.038 \pm 0.001$ & $0.050 \pm 0.001$ \\
\hline & 10 & $3258 \pm 68$ & $2069 \pm 42$ & $0.037 \pm 0.001$ & $0.050 \pm 0.001$ \\
\hline & 12 & $3279 \pm 68$ & $2081 \pm 42$ & $0.035 \pm 0.001$ & $0.050 \pm 0.001$ \\
\hline & 15 & $3310 \pm 69$ & $2080 \pm 43$ & $0.032 \pm 0.001$ & $0.049 \pm 0.001$ \\
\hline & 20 & $3352 \pm 70$ & $2088 \pm 43$ & $0.031 \pm 0.001$ & $0.047 \pm 0.001$ \\
\hline & 25 & $3374 \pm 71$ & $2101 \pm 43$ & $0.029 \pm 0.001$ & $0.047 \pm 0.001$ \\
\hline & 30 & $3395 \pm 72$ & $2109 \pm 43$ & $0.027 \pm 0.001$ & $0.048 \pm 0.001$ \\
\hline
\end{tabular}


perpendicular $\left(90^{\circ}\right)$ to the wave path, except for the P-waves in samples WUK47B and WUK70, in which the P-waves are attenuated mostly at $45^{\circ}$. This attenuation is partly due to the scattering on the different layers, and it is partly due to the intrinsic attenuation (viscous friction due to wave-induced local flow in cracks and fractures or grain-to-grain friction). Strong initial variations in attenuation values at low stress values can be explained by the nonperfect acoustic coupling between the samples and the aluminum buffers.
It is clear that an increase in axial compressive stress tends to decrease the attenuation as cracks and fissures are closed. These cracks and fractures contribute to the intrinsic attenuation so that the difference between the initial zero loading stage and the final ultimate loading stage is an indication for the intrinsic attenuation. Apparently, in samples WUK47B and WUK70, there are more randomly oriented cracks and fractures present that are closed as the axial loading is increased and the sample is compressed. The re-

Table 3. WUK70: measured $V_{\mathrm{P}}, V_{\mathrm{S}}, Q_{\mathrm{P}}^{-1}$, and $Q_{\mathrm{S}}^{-1}$ as a function of axial stress. $\mathrm{P}$ (or $\mathrm{S}$ ) indicates for stress applied during P- (or S)-wave measurement.

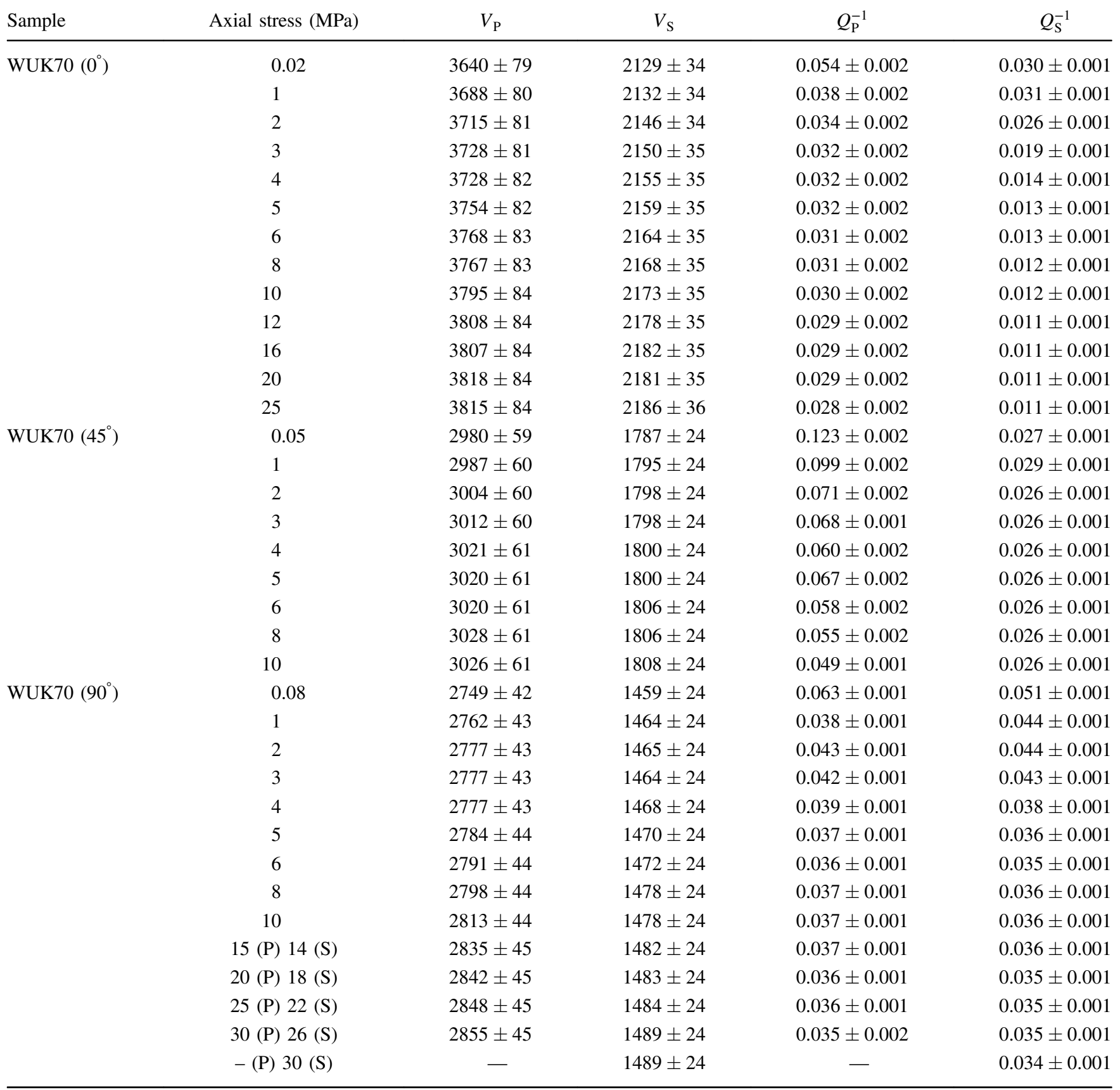


maining attenuation can then be attributed to the layer scattering. In this respect, one can intuitively understand that this scattering mechanism is most important for layers that are perpendicular to
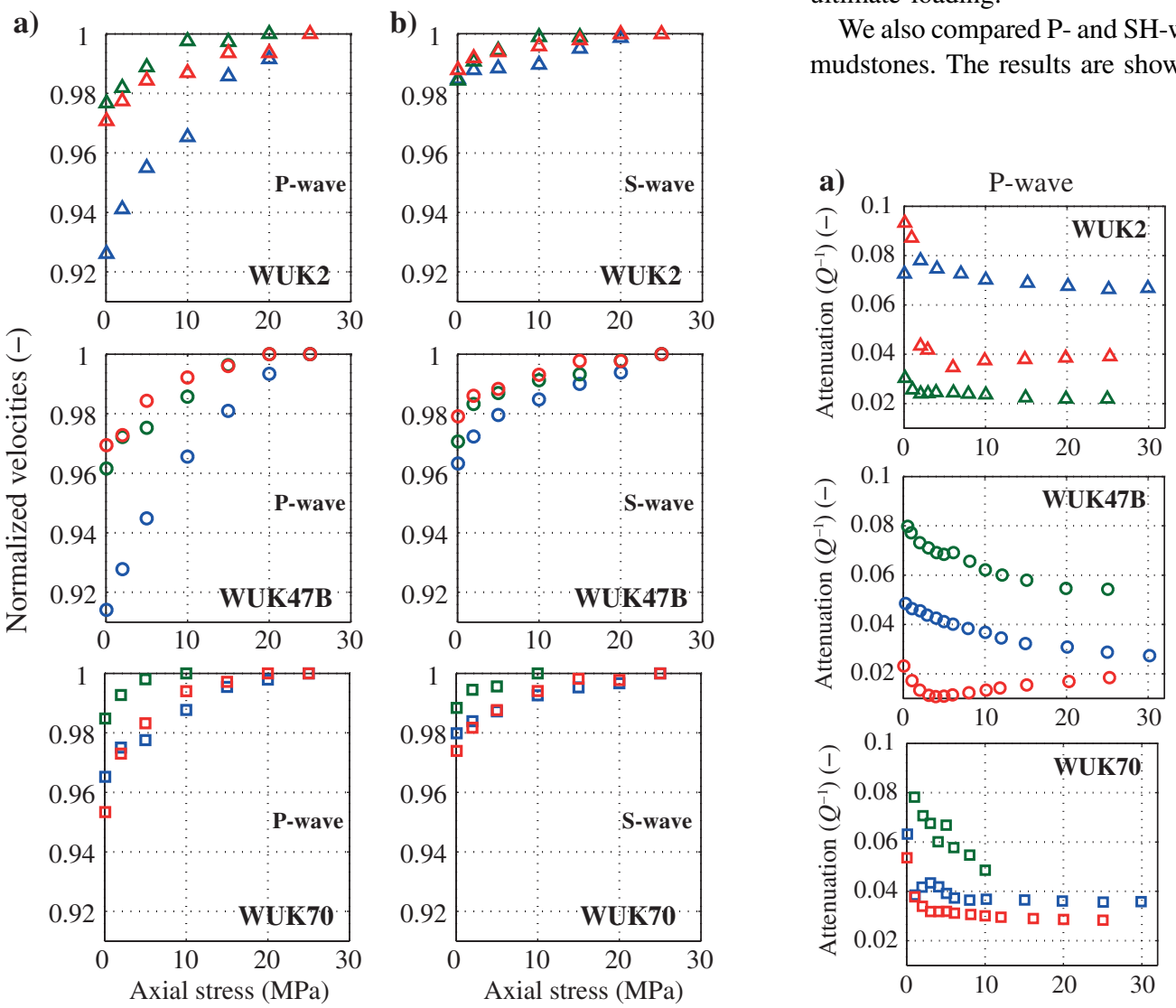

Figure 6. Normalized (a) P- and (b) S-wave velocities as a function of axial stress in blocks WUK2 (triangles), WUK47B (circles), and WUK70 (squares). The blue, green, and red colors correspond to cylindrical samples perpendicular $\left(90^{\circ}\right), 45^{\circ}$, and parallel $\left(0^{\circ}\right)$ to the bedding, respectively.
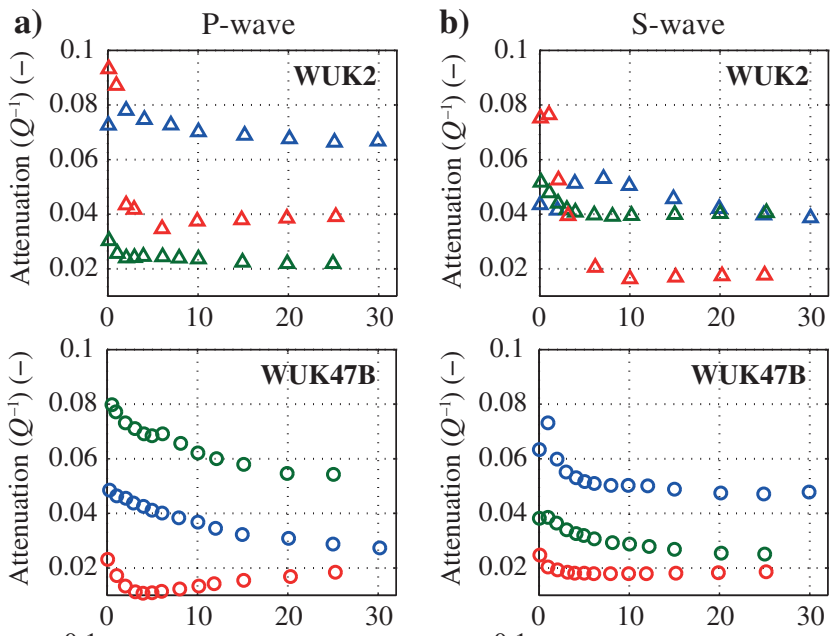

the propagating acoustic wave $\left(90^{\circ}\right.$ samples). It is also clear from Figure 8 that the ultimate loading stage for sample WUK47B was not yet reached, and that it is very well possible that the $90^{\circ} \mathrm{P}$-wave attenuation is larger than all others for sample WUK70 as well, at ultimate loading.

We also compared $\mathrm{P}$ - and $\mathrm{SH}$-wave attenuation obtained from our mudstones. The results are shown in Figure 9. It can be seen that
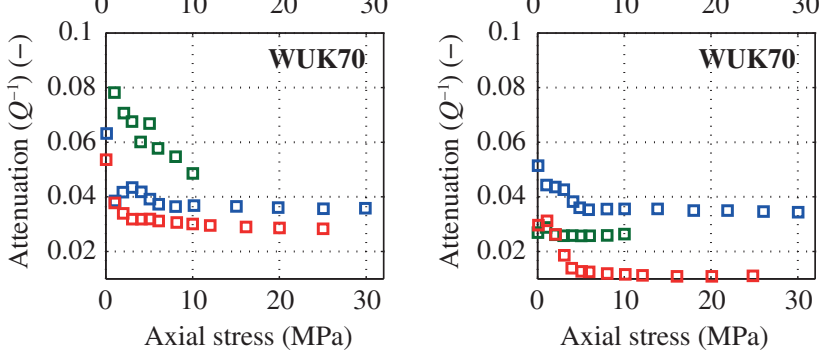
stress in blocks WUK2 (triangles), WUK47B (circles), and WUK70 (squares). The blue, green, and red colors correspond to samples $90^{\circ}, 45^{\circ}$, and $0^{\circ}$, respectively.
Figure 8. ( $\mathrm{a}$ and $\mathrm{b}$ ) Attenuation measurements as a function of axial
Figure 7. Dynamic Young's moduli and Poisson's ratios as a function of axial stress in blocks WUK2 (triangles), WUK47B (circles), and WUK70 (squares).
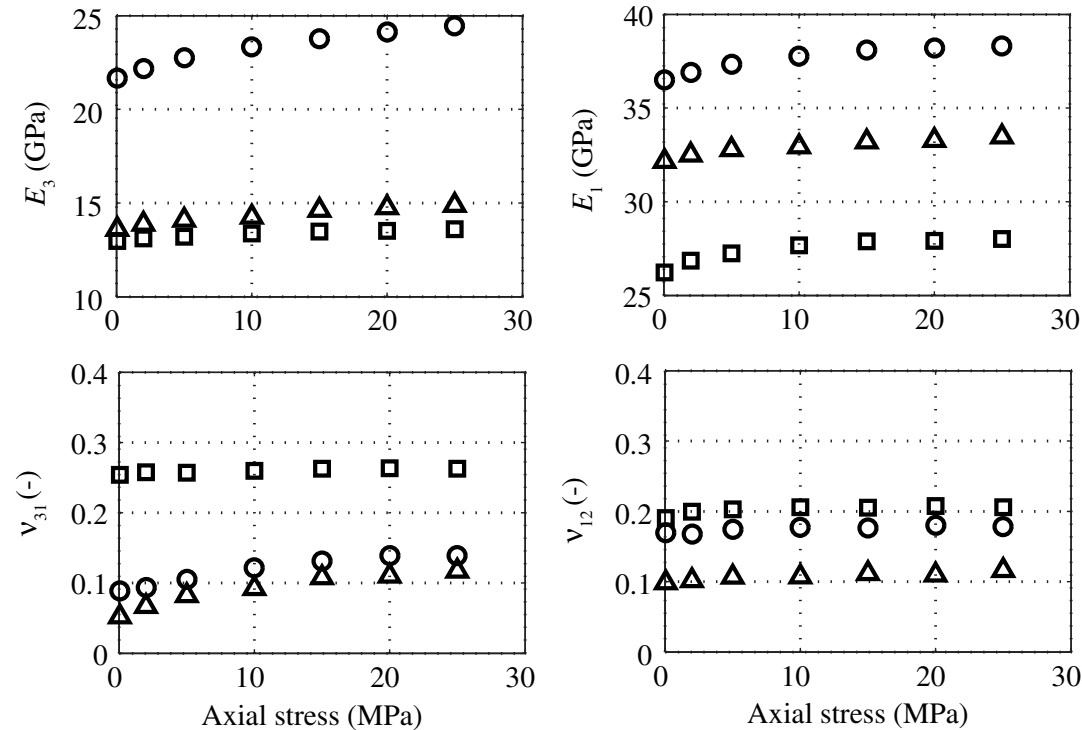
$\mathrm{P}$-waves tend to attenuate more than $\mathrm{SH}$-waves, consistent with the theoretical prediction based on the conceptual model of Winkler and Nur (1979) for partially saturated rocks. The opposite behavior observed in the samples (WUK2 at $45^{\circ}$ and WUK47B at $90^{\circ}$ ) can be related in the same way as mentioned above to more intrinsic attenuation caused by the presence of more cracks and fractures in the sample. To relate seismic attenuation to shale properties (e.g., permeability, porosity among others), one should subtract scattering attenuation from effective attenuation and look at the

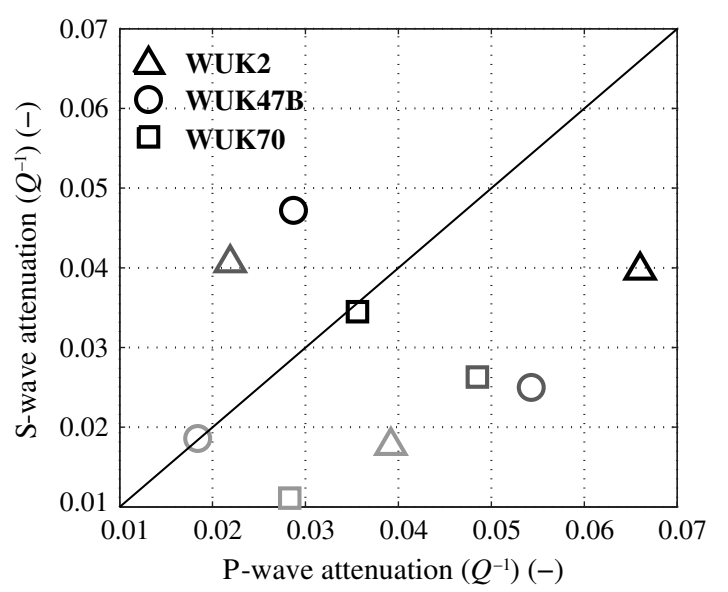

Figure 9. The $Q_{\mathrm{S}}^{-1}$ versus $Q_{\mathrm{P}}^{-1}$ at $25 \mathrm{MPa}$ axial stress in blocks WUK2 (triangles), WUK47B (circles), and WUK70 (squares). The black, dark gray, and light gray colors correspond to the samples at $90^{\circ}, 45^{\circ}$, and $0^{\circ}$, respectively.
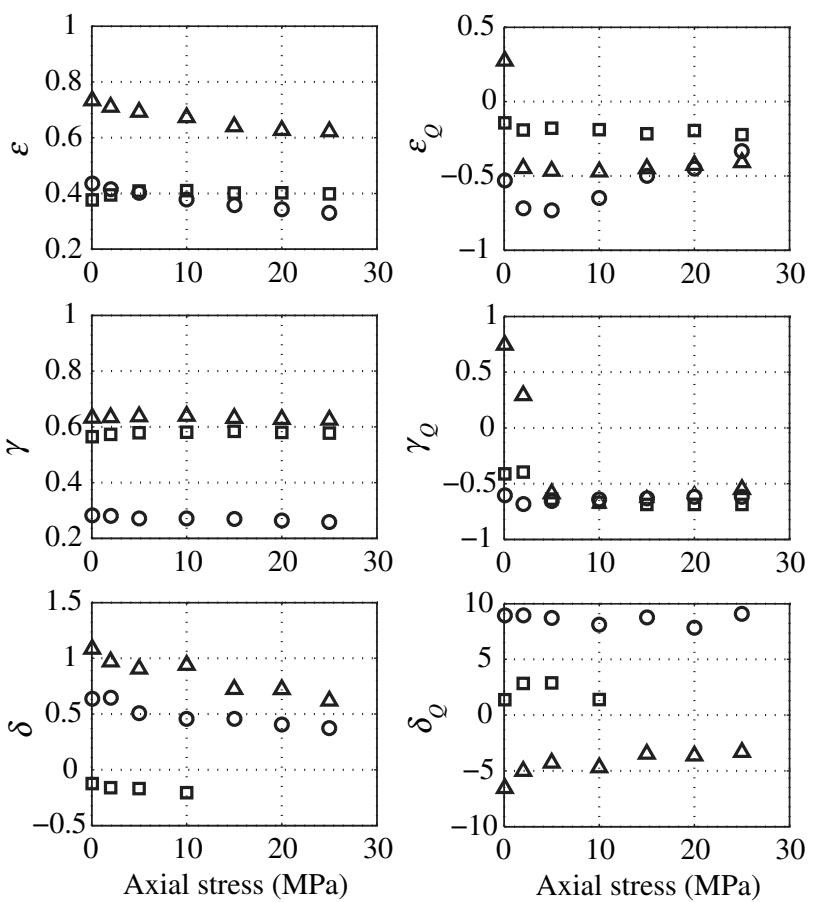

Figure 10. Velocity $(\epsilon, \gamma$, and $\delta)$ and attenuation $\left(\epsilon_{Q}, \gamma_{Q}\right.$, and $\left.\delta_{Q}\right)$ anisotropy parameters versus axial stress of blocks WUK2 (triangles), WUK47B (circles), and WUK70 (squares). sample's intrinsic attenuation. Thus, by means of rock physics models, one can invert for shale properties provided that the loss mechanism responsible for seismic attenuation is known. No attempts were made to quantify scattering attenuation in this study.

Finally, we calculated the velocity and attenuation anisotropy parameters using equations A-8-A-10 and A-14-A-16. The results are shown in Figure 10. Examination of the results in Figure 10 shows that, in general, attenuation and velocity anisotropy (expressed in terms of $\epsilon, \gamma, \delta, \epsilon_{Q}, \gamma_{Q}$, and $\delta_{Q}$ ) are strong and sensitive to stress and lithology. The changes of velocity and anisotropy with increasing stress are primarily caused by crack closure mechanisms, and at the highest stresses the changes of velocity and anisotropy become independent of the stress magnitude. As a consequence, at deeper subsurface conditions where most cracks are considered closed, it is expected that velocity and attenuation are less sensitive to the stress magnitude. The degree of seismic anisotropy can be as large as $70 \%$ (see the values of $\gamma, \epsilon, \gamma_{Q}$, and $\epsilon_{Q}$ in Figure 10). Similar to this study, large velocity and attenuation anisotropy values are reported by Yin (1992) and Best et al. (2007). One can see that the changes of P-wave velocity anisotropy with applied stress are more pronounced than those found from $\mathrm{SH}$-waves (compare $\epsilon$ and $\gamma$ in Figure 10). This can be explained by the closure of microcracks and compliant pores, which affects P-waves more (see Figure 6). This is a direct observation of changes in Thomsen's parameters due to rock property changes (e.g., crack density, porosity, and permeability). In case of attenuation anisotropy, the behavior of $\epsilon_{Q}, \gamma_{Q}$, and $\delta_{Q}$ is more complicated, and that makes interpretation more difficult. The changes in attenuation anisotropy with applied stress are more dynamic compared with velocity anisotropy, which is indicative of higher sensitivity of attenuation to rock property changes.

We should note here that this is an apparent velocity and attenuation anisotropy that can be different from the intrinsic anisotropy. For example, applied uniaxial stress only (this experiment) may cause an extra stress-induced anisotropy. In the future, one may need to conduct similar experiments but under confining pressures. Nevertheless, the results presented in this paper are valid for comparative purposes.

\section{CONCLUSIONS}

Ultrasonic experiments between a 0.3 and $1 \mathrm{MHz}$ range have been conducted to study ultrasonic anisotropy in dry Whitby Mudstone outcrop samples as a function of applied axial stress. The experimental results showed that the degree of ultrasonic anisotropy can be as large as $70 \%$. The change of velocity anisotropy of P-waves with applied stress was more apparent than those found from $\mathrm{S}$-waves. The changes in attenuation anisotropy with applied stress were more dynamic with respect to the velocity anisotropy supporting the validity of higher sensitivity of attenuation to rock property changes.

The rate of velocity increase for Whitby Mudstone (or decrease for $Q^{-1}$ ) was high at low pressures $(<10 \mathrm{MPa})$, and it was nearly constant at high pressures. The P-waves were found to be more sensitive to pore (and/or microcracks) closure than were the $\mathrm{S}$-waves. Attenuation for P- and S-waves was found to be generally larger in horizontally layered $\left(90^{\circ}\right)$ samples with a stress direction perpendicular to the layering than in the $0^{\circ}$ samples in which the layering and stress directions are parallel. The change in attenuation due to crack closure at increasing stresses is attributed to the intrin- 
sic attenuation of the mudstone samples. The remaining attenuation is then a consequence of the scattering attenuation between the layers in the mudstones. The measured anisotropy data can be useful for understanding and/or validating loss mechanisms responsible for seismic attenuation and can be of use in developing new theoretical rock models that translate seismic data to intrinsic rock property anisotropy.

\section{ACKNOWLEDGMENTS}

Funding from the Dutch Upstream Gas top-sector initiative (project no. TKIG01020) and our industry partners EBN, GDF Suez, Wintershall, Total, and Baker Hughes is acknowledged. We are also indebted to K. Heller, A. Mulder, and D. Delforterie for their technical support during the experiments. The constructive reviews by three anonymous reviewers are greatly appreciated.

\section{APPENDIX A}

\section{ANISOTROPY IN DISSIPATIVE VTI MEDIA}

\section{Velocity anisotropy}

Many shale samples can be described well by considering them as being TI with an axis of rotational symmetry aligned perpendicular to the bedding plane. The nondissipative TI medium has five independent elastic stiffnesses $C_{i j}$. Taking the axis $x_{3}$ as the axis with the rotational symmetry, the stress-strain relation can be written (Mavko et al., 2009) as follows:

$$
\left[\begin{array}{l}
\sigma_{11} \\
\sigma_{22} \\
\sigma_{33} \\
\sigma_{31} \\
\sigma_{23} \\
\sigma_{12}
\end{array}\right]=\left[\begin{array}{cccccc}
C_{11} & C_{12} & C_{13} & 0 & 0 & 0 \\
C_{12} & C_{11} & C_{13} & 0 & 0 & 0 \\
C_{13} & C_{13} & C_{33} & 0 & 0 & 0 \\
0 & 0 & 0 & C_{55} & 0 & 0 \\
0 & 0 & 0 & 0 & C_{55} & 0 \\
0 & 0 & 0 & 0 & 0 & C_{66}
\end{array}\right]\left[\begin{array}{l}
e_{11} \\
e_{22} \\
e_{33} \\
e_{31} \\
e_{23} \\
e_{12}
\end{array}\right],
$$

where $\sigma_{i j}$ and $e_{i j}$ denote stress and strain, respectively, and $C_{11}=C_{22}, C_{12}=C_{21}, C_{13}=C_{31}=C_{23}=C_{32}, C_{44}=C_{55}$. Finally, $C_{66}=\left(C_{11}-C_{12}\right) / 2$. These elastic stiffness coefficients $C_{i j}$ can be obtained from the measured $\mathrm{P}$ - and $\mathrm{S}$-wave velocities and the density $\rho$ of the sample as follows:

$$
\begin{gathered}
C_{11}=\rho V_{\mathrm{P}}^{2}\left(0^{\circ}\right), \\
C_{12}=C_{11}-2 \rho V_{\mathrm{SH}}^{2}\left(0^{\circ}\right), \\
C_{33}=\rho V_{\mathrm{P}}^{2}\left(90^{\circ}\right), \\
C_{44}=\rho V_{\mathrm{SH}}^{2}\left(90^{\circ}\right), \\
C_{13}=-C_{44}+\left(4 \rho^{2} V_{\mathrm{P}}^{4}\left(45^{\circ}\right)\right. \\
-2 \rho V_{\mathrm{P}}^{2}\left(45^{\circ}\right)\left(C_{11}+C_{33}+2 C_{44}\right) \\
\left.+\left(C_{11}+C_{44}\right)\left(C_{33}+C_{44}\right)\right)^{1 / 2},
\end{gathered}
$$

$$
C_{66}=\left(C_{11}-C_{12}\right) / 2 \text {, }
$$

where $V_{\mathrm{P}}\left(90^{\circ}\right), V_{\mathrm{P}}\left(0^{\circ}\right)$, and $V_{\mathrm{P}}\left(45^{\circ}\right)$ are the seismic velocities of the $\mathrm{P}$-wave propagating, respectively, orthogonal, parallel, and at $45^{\circ}$ to the bedding. The $V_{\mathrm{SH}}\left(90^{\circ}\right)$ is the velocity of the S-wave propagating orthogonal to the bedding, and the plane of polarization is in the $x_{1}-x_{3}$ (or $\left.x_{2}-x_{3}\right)$ plane. The $V_{\mathrm{SH}}\left(0^{\circ}\right)$ is the velocity of the $\mathrm{S}$-wave propagating parallel to the bedding, and the plane of polarization is in the symmetry $\left(x_{1}-x_{2}\right)$ plane. The bulk density $(\rho)$ was measured for each sample prior experiment (see text above). Thus, by estimating $V_{\mathrm{P}}\left(90^{\circ}\right), V_{\mathrm{P}}\left(0^{\circ}\right), V_{\mathrm{P}}\left(45^{\circ}\right), V_{\mathrm{SH}}\left(90^{\circ}\right), V_{\mathrm{SH}}\left(0^{\circ}\right)$, and $\rho$, the $C_{i j}$ coefficients can be calculated.

The so-called Thomsen's notation (Thomsen, 1986) is commonly used to describe the elastic anisotropy of a geomaterial. These are defined by the following equations:

$$
\begin{gathered}
\epsilon=\frac{C_{11}-C_{33}}{2 C_{33}}, \\
\gamma=\frac{C_{66}-C_{55}}{2 C_{55}}, \\
\delta=\frac{\left(C_{13}+C_{55}\right)^{2}-\left(C_{33}-C_{55}\right)^{2}}{2 C_{33}\left(C_{33}-C_{55}\right)} .
\end{gathered}
$$

Finally, substituting $C_{i j}$ in equations A-8 $-\mathrm{A}-10$, one can obtain the anisotropic (Thomsen's) parameters.

\section{Attenuation anisotropy}

According to Zhu and Tsvankin (2006), the anisotropic quality factor can be described by matrix elements $Q_{i j}$ and for the case of TI media with TI attenuation can be written as follows:

$$
\mathbf{Q}=\left[\begin{array}{cccccc}
Q_{11} & Q_{12} & Q_{13} & 0 & 0 & 0 \\
Q_{12} & Q_{11} & Q_{13} & 0 & 0 & 0 \\
Q_{13} & Q_{13} & Q_{33} & 0 & 0 & 0 \\
0 & 0 & 0 & Q_{55} & 0 & 0 \\
0 & 0 & 0 & 0 & Q_{55} & 0 \\
0 & 0 & 0 & 0 & 0 & Q_{66}
\end{array}\right]
$$

where

$$
Q_{12}=Q_{11} \frac{C_{11}-2 C_{66}}{C_{11}-2 C_{66} \frac{Q_{11}}{Q_{66}}} .
$$

The axis orientations $\left(x_{1}, x_{2}, x_{3}\right)$ with respect to the bedding are the same as in the previous velocity anisotropy section of the Appendix A. In terms of the complex stiffness coefficients, the $Q$-factor matrix is given by

$$
Q_{i j} \equiv \frac{C_{i j}}{C_{i j}^{I}},
$$

where $C_{i j}$ and $C_{i j}^{I}$ are the real and the imaginary parts, respectively, of the stiffness coefficient $\hat{C}_{i j}=C_{i j}+i C_{i j}^{I} ; C_{i j}$ and $C_{i j}^{I}$ are the Hilbert pairs and related through the Kramers-Kronig relationship. Note that there is no summation over repeated indices in equa- 
tion A-13. To characterize TI attenuation, Zhu and Tsvankin (2006) follow the idea of the Thomsen notation for velocity anisotropy (see equations A-8-A-10) and derive three dimensionless parameters to quantify attenuation anisotropy as follows:

$$
\begin{gathered}
\epsilon_{\mathrm{Q}}=\frac{Q_{33}-Q_{11}}{Q_{11}}, \\
\gamma_{\mathrm{Q}}=\frac{Q_{55}-Q_{66}}{Q_{66}}, \\
\delta_{\mathrm{Q}}=\frac{\frac{Q_{33}-Q_{55}}{Q_{55}} C_{55} \frac{\left(C_{13}+C_{33}\right)^{2}}{\left(C_{33}-C_{55}\right)}+2 \frac{Q_{33}-Q_{13}}{Q_{13}} C_{13}\left(C_{13}+C_{55}\right)}{C_{33}\left(C_{33}-C_{55}\right)} .
\end{gathered}
$$

To derive these expressions, weak attenuation anisotropy is assumed, where the real and imaginary parts of the wave vector are parallel to one another. The parameters $\epsilon_{Q}$ and $\gamma_{Q}$ quantify the difference between the horizontal- and vertical-attenuation coefficients of $\mathrm{P}$ - and $\mathrm{SH}$-waves, respectively, and $\delta_{Q}$ is defined through the second derivative of the $\mathrm{P}$-wave attenuation coefficient in the symmetry direction.

Four out of five elements in the $\mathbf{Q}$ matrix (equation A-11) can be obtained directly from $\mathrm{P}$ - and $\mathrm{SH}$-wave attenuation measurements on our three cylindrical samples with different axis orientations with respect to the bedding (see Figure 1):

$$
\begin{gathered}
Q_{11}=Q_{\mathrm{P}}\left(0^{0}\right), \\
Q_{33}=Q_{\mathrm{P}}\left(90^{0}\right), \\
Q_{44}=Q_{55}=Q_{\mathrm{SH}}\left(90^{0}\right), \\
Q_{66}=Q_{\mathrm{SH}}\left(0^{0}\right) .
\end{gathered}
$$

To calculate $Q_{13}$, we follow the definition of the $\mathbf{Q}$ matrix (equation A-13). Thus, $Q_{13}$ can be obtained from the ratio of the real and the imaginary part of elastic stiffness coefficient $\hat{C}_{13}$ :

$$
Q_{13} \equiv \frac{C_{13}}{C_{13}^{I}},
$$

where $C_{13}^{I}$ can be calculated analogous (assuming the similarity between $C_{i j}$ and $C_{i j}^{I}$ structure) to equation A-6 as

$$
\begin{aligned}
C_{13}^{I}= & -\frac{C_{44}}{Q_{44}}+\left(4 \frac{\rho^{2} V_{\mathrm{P}}^{4}\left(45^{\circ}\right)}{Q_{\mathrm{P}}^{2}\left(45^{\circ}\right)}\right. \\
& -2 \frac{\rho V_{\mathrm{P}}^{2}\left(45^{\circ}\right)}{Q_{\mathrm{P}}\left(45^{\circ}\right)}\left(\frac{C_{11}}{Q_{11}}+\frac{C_{33}}{Q_{33}}+2 \frac{C_{44}}{Q_{44}}\right) \\
& \left.+\left(\frac{C_{11}}{Q_{11}}+\frac{C_{44}}{Q_{44}}\right)\left(\frac{C_{33}}{Q_{33}}+\frac{C_{44}}{Q_{44}}\right)\right)^{1 / 2} .
\end{aligned}
$$

Combining equations A-6, A-21, and A-22, one can calculate the $Q_{13}$ element of the $\mathbf{Q}$ matrix.

\section{Young's moduli and Poisson's ratios}

For a TI medium, one can get two independent Young's moduli: $E_{1}=E_{2}$ and $E_{3}$, and two Poisson's ratios: $\nu_{21}=\nu_{12}$ and $\nu_{31}=\nu_{13}$; $E_{i}$ corresponds to the axis $x_{i}$, and $\nu_{i j}$ relates elastic strain in $x_{j}$ direction to stress applied in $x_{i}$ direction. The Young's moduli and Poisson's ratios can be calculated from the following equations:

$$
\begin{gathered}
\nu_{12}=\frac{\left(V_{\mathrm{P}}\left(0^{\circ}\right) / V_{\mathrm{SH}}\left(0^{\circ}\right)\right)^{2}-2}{2\left(V_{\mathrm{P}}\left(0^{\circ}\right) / V_{\mathrm{SH}}\left(0^{\circ}\right)\right)^{2}-1}, \\
\nu_{31}=\frac{\left(V_{\mathrm{P}}\left(90^{\circ}\right) / V_{\mathrm{SH}}\left(90^{\circ}\right)\right)^{2}-2}{2\left(V_{\mathrm{P}}\left(90^{\circ}\right) / V_{\mathrm{SH}}\left(90^{\circ}\right)\right)^{2}-1}, \\
E_{1}=2 C_{66}\left(1+\nu_{12}\right), \\
E_{3}=2 C_{44}\left(1+\nu_{31}\right) .
\end{gathered}
$$

\section{REFERENCES}

Best, A. I., J. Sothcott, and C. McCann, 2007, A laboratory study of seismic velocity and attenuation anisotropy in near-surface sedimentary rocks: Geophysical Prospecting, 55, 609-625, doi: 10.1111/j.1365-2478.2007 .00642.x.

Carcione, J. M., J. E. Santos, and S. Picotti, 2012, Fracture-induced anisotropic attenuation: Rock Mechanics and Rock Engineering, 45, 929-942 10.1007\%2Fs00603-012-0237-y\#.

Chichinina, T., S. Sabinin, and G. Ronquillo-Jarillo, 2006, QVOA analysis: P-wave attenuation anisotropy for fracture characterization: Geophysics, 71, no. 3, C37-C48, doi: 10.1190/1.2194531.

Ghadeer, S. G., and J. Macquaker, 2012, The role of event beds in the preservation of organic carbon in fine-grained sediments: Analysis of the sedimentological processes operating during deposition of the Whitby Mudstone Formation (Toarcian, Lower Jurassic) preserved in northeast England: Marine and Petroleum Geology, 35, 309-320, doi: 10.1016/j .marpetgeo.2012.01.001.

Hornby, B., 1998, Experimental laboratory determination of the dynamic elastic properties of wet, drained shales: Journal of Geophysical Research, 103, 29945-29964, doi: 10.1029/97JB02380.

Houben, M., A. Barnhoorn, M. Drury, C. Peach, and C. Spiers, 2014, Microstructural investigation of the Whitby Mudstone (UK) as an analog for Posidonia Shale (NL): 76th Annual International Conference and Exhibition, EAGE, Extended Abstracts, doi: 10.3997/2214-4609.20141056.

Imber, J., H. Armstrong, S. S. Clancy, S. S. Daniels, L. Herringshaw, K. McCaffrey, J. Rodrigues, J. Trabucho-Alexandre, and C. Warren, 2014, Natural fractures in a United Kingdom shale reservoir analog, Cleveland Basin, northeast England: AAPG Bulletin, 98, 2411-2437, doi: $10.1306 / 07141413144$.

Jones, J., and H. Wang, 1981, Ultrasonic velocities in Cretaceous shales from the Williston Basin: Geophysics, 46, 288-297, doi: 10.1190/1 .1441199 .

Lucet, N., and B. Zinszner, 1992, Effects of heterogeneities and anisotropy on sonic and ultrasonic attenuation in rocks: Geophysics, 57, 1018-1026, doi: 10.1190/1.1443313.

Mavko, G., T. Mukerji, and J. Dvorkin, 2009, The rock physics handbook, 2nd ed.: Cambridge University Press.

McArthur, J. M., T. J. Algeo, B. van de Schootbrugge, Q. Li, and R. J. Howarth, 2008, Basinal restriction, black shales, re-os dating, and the Early Toarcian (Jurassic) oceanic anoxic event: Paleoceanography, 23, PA4217, doi: 10.1029/2008PA001607.

Piane, C. D., J. Sarout, C. Madonna, E. H. Saenger, D. Dewhurst, and M. Raven, 2014, Frequency-dependent seismic attenuation in shales: Experimental results and theoretical analysis: Geophysical Journal International, 198, 504-515, doi: 10.1093/gji/ggu148.

Pyrak-Nolte, L. J., L. Myer, and N. Cook, 1990, Transmission of seismic waves across single natural fractures: Journal of Geophysical Research, 95, 8617-8638, doi: 10.1029/JB095iB06p08617.

Rasolofosaon, P. N. J., and B. E. Zinszner, 2002, Comparison between permeability anisotropy and elasticity anisotropy of reservoir rocks: Geophysics, 67, 230-240, doi: 10.1190/1.1451647.

Rubino, J. G., T. Muller, L. Guarracino, M. Milani, and K. Holliger, 2014, Seismoacoustic signatures of fracture connectivity: Journal of Geophysical Research, 119, 2252-2271. 
Sayers, C. M., 1994, The elastic anisotropy of shales: Journal of Geophysical Research, 99, 767-774, doi: 10.1029/93JB02579.

Sayers, C. M., 2013, The effect of anisotropy of the Young's moduli and Poisson's ratios of shales: Geophysical Prospecting, 61, 416-426, doi: 10.1111/j.1365-2478.2012.01130.x.

Thomsen, L., 1986, Weak elastic anisotropy: Geophysics, 51, 1954-1966, doi: 10.1190/1.1442051.

Toksöz, M., D. Johnston, and A. Timur, 1979, Attenuation of seismic waves in dry and saturated rocks: I-Laboratory measurements: Geophysics, $\mathbf{4 4}$, 681-690, doi: 10.1190/1.1440969.

Vernik, L., and X. Liu, 1997, Velocity anisotropy in shales: A petrophysical study: Geophysics, 62, 521-532, doi: 10.1190/1.1444162.
Wang, Z, 2002, Seismic anisotropy in sedimentary rocks: Part II - Laboratory data: Geophysics, 67, 1423-1440, doi: 10.1190/1.1512743.

Winkler, K., and A. Nur, 1979, Pore fluids and seismic attenuation in rocks: Geophysical Research Letters, 6, 1-4, doi: 10.1029/GL006i001p00001.

Yin, H., 1992, Acoustic velocity and attenuation of rocks: Isotropy, intrinsic anisotropy, and stress induced anisotropy: Ph.D. thesis, Stanford University.

Zemanek, J., and I. Rudnick, 1961, Attenuation and dispersion of elastic waves in a cylindrical bar: Journal of the Acoustical Society of America, 33, 1283-1288, doi: 10.1121/1.1908417.

Zhu, Y., and I. Tsvankin, 2006, Plane-wave propagation in attenuative transversely isotropic media: Geophysics, 71, no. 2, T17-T30, doi: 10.1190/1 .2187792 . 